\title{
Microrépartition des algues épilithiques sur les cailloux d'un torrent Corse, le Rizzanèse
}

\author{
A. Cazaubon 1 \\ M. Loudiki 1
}

Mots clés : Algues épilithiques, microrépartition, caillou, torrent, Corse du Sud, traitement des données, méthodologie.

L'étude de la composition et de la structure du peuplement algal épilithique, en divers points d'un même caillou, fait apparaitre deux ensembles:

1) la face orientée vers l'aval et le centre de la face supérieure:

2) la face amont et le centre de la face inférieure.

L'analyse statistique montre que le peuplement de la face supérieure, du fait de sa plus grande stabilité, est le plus représentatif de la communauté algale des substrats caillouteux.

Microdistribution of epilithic algae on the stones of a Corsican stream, the Rizzanèse.

Keywords : Epilithic algae, microdistribution, pebble, torrent, South Corsica, statistical analysis, methodology.

A study of the composition and structure of epilithic algal populations at several places on the same pebble revealed iwo groups :

1) on the downstream face and the center of the upper face of the stone ;

2) on the upstream face and the center of the lower face of the stone.

A statistical analysis showed that the population on the upper face, because of it s greatest stability, was the most representative of the algal community of stony substrata.

\section{Introduction}

Au cours de recherches sur les algues épilithiques de rivières de montagne, nous nous sommes heurtés d'emblée aux problèmes de l'échantillonnage de ces peuplements. A notre connaissance, il n'existe pas de méthodes de prélèvement satisfaisantes pour ce type de biotope, caractérisé par des écoulements rapides et par l'hétérogénéité des matériaux du fond. Le cours d'eau sur lequel l'étude a été réalisée, le Rizzanèse, est situé en Corse du Sud. Il est représentatif des cours d'eau de montagne de la

1. Laboratoire de Biologie Animale et Ecologie, Faculté des Sciences de St. Jerôme, nue Henri-Poincaré, 13397 Marseille Cédex 13. zone méditerranéenne du fait de particularités physiques liées à la fois au climat et à la géomorphologie des bassins versants.

La vitesse du courant et les variations de débit ne permettent pas l'utilisation des supports artificiels pour l'échantillonnage des algues benthiques. Nous avons dû réaliser l'étude par échantillonnage direct sur le substrat naturel représenté essentiellement par des cailloux.

La présente étude se propose de développer les points suivants :

- mettre en évidence le rôle du courant sur la micro-distribution des algues à la surface des cailloux ;

- tester la fiabilité de la méthode d'échantillonnage retenue et décrite ici. 
D'autres données, obtenues avec la même technique de récolte, mais dans des cours d'eau à conditions hydrologiques différentes, viendront en complément de ce premier essai.

\section{1. - Le milieu}

\section{1. - Conditions écologiques générales}

Le Rizzanèse, rivière de montagne de la Corse du Sud, prend sa source à $1800 \mathrm{~m}$ d'altitude et se jette, après un parcours d'environ $53 \mathrm{~km}$, dans la Méditerranée, à proximité de la ville de Propriano sur la côte occidentale. Elle draine un bassin versant de $396 \mathrm{~km}^{2}$; sa pente moyenne est de $4 \%$.

La station prospectée est située sur le cours axial (altitude $: 300 \mathrm{~m}$ ), à $23 \mathrm{~km}$ de la source, près du village de Serra di Scopamene. A ce niveau, le lit de la rivière, large d'environ 15 à $20 \mathrm{~m}$, est formé de blocs, de galets et de cailloux de nature cristalline (granite, diorite et gabbro). Les macrophytes sont absents ; la ripisilve est dense, dominée par le peuplier blanc et l'aulne glutineux.

Les prélèvements de la microflore benthique ont été effectués en mars 1984, époque succédant cette année-là, à une période de stabilité hydrologique assez exceptionnelle pour ce type de cours d'eau (en effet, les précipitations ont été très rares et peu abondantes pendant l'hiver 84).

\section{2. - Caractères physico-chtmiques des eaux}

- Descripteurs physiques

- hauteur d'eau : $45 \mathrm{~cm}$

- température de l'eau : $5^{\circ} \mathrm{C}$

- $\mathrm{pH}: 7,8$

- conductivité : $108 \mu \mathrm{s} / \mathrm{cm}$

- oxygène dissous : $11,3 \mathrm{mg} / \mathrm{l}$

(\% de saturation $99 \%$ )

- Descripteurs chimiques

Cations

- Calcium : $5,5 \mathrm{mg} / \mathrm{l}$

- Magnesium : 2,9

- Sodium : 7,1

- Potassium : 0,6

- Azote ammoniacal : 0,00

Anions

- Bicarbonates : $19 \mathrm{mg} / \mathrm{l}$

- Chlorures : 15

- Sulfates $\quad: 0,00$
- Silice : $\mathbf{4 , 5}$

- Azote nitreux $: 0,00$

- Azote nitrique : 1,15

- Orthophosphates : 0,00

Le chimisme de l'eau de cette rivière est tout à fait caractéristique des cours d'eau de la Corse cristalline, avec une remarquable pauvreté en éléments dissous. La forte pente provoque un brassage permanent de l'eau assurant ainsi sa saturation en oxygène. L'absence totale des nitrites, de l'ammoniaque, des orthophosphates et les très faibles valeurs de la $\mathrm{DB} 05$ et de l'oxydation $\left(0,3 \mathrm{mg} / \mathrm{l}\right.$ d' $\mathrm{O}_{2}$ chacune) sont indicatrices d'une eau totalement exempte de pollution.

\section{2. - Méthode d'étude du peuplement algal benthique}

D'une manière générale, les différentes méthodes d'échantillonnage d'algues sont souvent critiquées au point que la valeur des résultats des prélèvements est contestée.

Certains auteurs ont prélevé directement sur substrats naturels. Douglas (1958) a utilisé un échantillonneur à siphon, Young (1945) une enceinte de capture, Descy (1976) une pompe à diatomées. D'autres ont préconisé l'utilisation de substrats artificiels tels que : plaque de verre (Castenholz 1961); diatomètre (Patrick 1973) ; plaque de plexiglass (Cooper \& Wilhm 1975) ; lame histologique (Cattaneo \& al 1975); feuille de polyéthylène (Cassan 1978; Galvin 1982 ; etc.).

Dans le cas d'eau courante profonde où la hauteur d'eau ne permet pas de prélever sur substrats naturels, la technique des supports artificiels est la seule utilisée. De nombreux chercheurs l'ont mise en ceuvre en milieux potamiques (Coste 1978), en rivière aménagées (Capblancq \& Cassan 1979) ou en secteur d'eau calme (Galvin \& Cazaubon 1983). L'utilisation de ces supports artificiels s'est révélée cependant inadéquate dans nos recherches car, les substrats que nous avons placés ont été emportés par des crues imprévisibles.

Dans ce type de cours d'eau de montagne, caractérisé par une faible profondeur et un écoulement rapide, les prélèvements de microflore nécessitent des techniques adaptées à l'hétérogénéité des éléments du substrat (depuis le sable jusqu'à la dalle 
rocheuse) et à des vitesses de courants élevées ivitesse, comprise entre 80 et $90 \mathrm{~cm} / \mathrm{s}$, correspondant a des courants rapides dans l'échelle de Berg).

Nous avons donc étudié la microflore benthique directement sur substrat naturel représenté essentiellement par des cailloux. Afin d'apprécier le degré de représentativité d'un prélèvement par rapport au peuplement périphytique présent dans la station, un test statistique (coefficient de variation et comparaison de moyennes) a été appliqué sur une série de 20 prélèvements effectués, simultanément, en mars 84 .

La surface du lit où se font les récoltes n'excède pas $4 \mathrm{~m}^{2}$. Elle se situe au milieu de la rivière en faciès rapide. La vitesse du courant était à peu près homogène $(80$ à $90 \mathrm{~cm} / \mathrm{s})$ sur la surface échantillonnée $\left(4 \mathrm{~m}^{2}\right)$. Nous avons prélevé 20 cailloux de même nature, de forme plus ou moins discoïdale, de taille comprise entre 5 et $15 \mathrm{~cm}$ de diamètre et de 1 à $4 \mathrm{~cm}$ de hauteur. Après avoir marqué la face amont de chaque caillou (orientation définie par rapport au sens du courant) nous les immergeons immédiatement dans un bocal d'eau formolée.

On a également prélevé deux autres cailloux qui nous paraissent présenter, pour le peuplement algal, des conditions d'habitat différentes : l'un, déprimé au milieu de sa surface supérieure et l'autre, abrité derrière un gros bloc.

En laboratoire, les échantillons d'algues sont obtenus par grattage au scalpel d'une surface de caillou de un $\mathrm{cm}^{2}$ puis montés directement entre lame et lamelle dans une goutte d'eau distillée. Les cellules algales sont déterminées et dénombrées ; les résultats sont donc exprimés en nombre de cellules par unité de surface $\left(\mathrm{cm}^{2}\right)$.

D'autres échantillons ont servi à la détermination des Diatomophycées ; pour ces algues, on élimine la matière organique par un traitement a $\mathrm{H}_{2} \mathrm{O}_{2}$ concentrée et $\mathrm{HCl}$ (méthode de Coste 1978).

Dans les rivières torrentielles, en plus de la diversité intrabiotopique, due à l'hétérogénéité des matériaux du lit, il faut aussi prendre en compte, dans le cas d'un peuplement algal épilithique, l'existence de conditions abiotiques qui s'établissent sur les différentes faces d'un caillou en fonction de leur exposition au courant. Pour cette raison, nous avons prélevé la microflore benthique d'un même caillou au niveau de quatre microhabitats différents de ce caillou (fig. 1) :

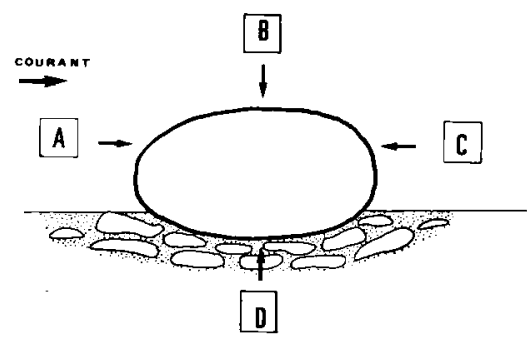

Fig. 1. Emplacement sur le caillou des aires d'échantillonnage.

- trois sur la face supérieure : 1 en amont (A), $I$ au centre (B) et $I$ en aval (C) ;

- un au centre de la face inférieure (D) ; (l'orientation amont-aval étant définie ici par rapport au sens de courant).

Pour la série des 20 cailloux, nous avons donc réalisé 80 prélèvements de substrat de $1 \mathrm{~cm}^{2}$ chacun. Les résultats de ces prélèvements (densité et richesse spécifique) ont été traités statistiquement (moyennes, écarts types et coefficients de variations).

\section{3. - Résultats et discussion}

\section{1. - Inventaire et composition du peuplement}

Au total 47 espèces et variétés ont été identifiées dans la station prospectée (Tableau I). Elles sont réparties en 5 classes d'algues, celle des Diatomophycées étant la mieux représentée avec 40 espèces. Les Chlorophycées comptent 4 espèces seulement ; les Chrysophycées, les Cyanophycées et les Rhodophycées enfin, ne sont représentées que par une seule espèce chacune.

Deux groupes se distinguent:

1) Un groupement principal constitué d'espèces traduisant la particularité du biotope étudié. Il s'agit de l'association Ceratoneito-Hydruretum rivulare, caractéristique des eaux froides et rapides des rivièress de haute montagne (Margalef 1956). 
Tableau I. Liste des espèces inventoriées sur les cailloux

\section{CHRYSOPHYCÉES}

Hydrurus foetidus VILL.

\section{DIATOMOPHYCÉES}

Achnanthes bioreti GERMAIN

$$
\begin{aligned}
& \text { lanceolata (BREB.) GRUN. var. lanceolata } \\
& \text { rostrata OSTRUP } \\
& \text { lapponica (HUST.) HUST. } \\
& \text { minutissima KUTZ. }
\end{aligned}
$$

Amphora pediculus (KUTZ.) GRUN

Cocconeis placentula EHR.

Cymbella affinis KUTZ.

minua HILSE ex RABH var. minuta

sinuata GREC.

tumidula GRUN

Denticula tenuis KUTZ.

Diatoma hiemale (ROTH) HEIB var. hiemale hiemale (ROTH) HEIB var, mesodon (EHR.) GRUN.

Epithemia adnata (KÜTZ.) BREB.

Eunotia pectinalis (O.F. MULLL) RABH. var. pectinalis

Fragilaria capucina DESM. var. lanceolata GRUN.

$$
\text { rumpens (KUTZ.) CARLSON }
$$

vaucheriae (KÜTZ.) PET.

vaucheriae (KUTZ.) PET, var, vaucheriae

Gomphonema angustafum (KÜTZ.) RABH. var. productum GRUN. dichotomum KÜTZ.

truncatum EHR. var. truncatum

Hannaea arcus (EHR.) PATR

Melosira varians AG.

Meridion circulare (GREV.) AG.

Navicula cryptocephala KUTZ. var. cryptocephala lanceolata EHR.

rhynchocephala KÜTZ.

tripunctata (O. MULL.) BORY

Nitzschia dissipata (KUTZ.) GRUN.

hantzschiana RABH.

linearis W. SMITH

palea (KUTZ.) W. SMITH

Pinnularia borealis $\mathrm{EHR}$. viridis (NITZ.) EHR. var. viridis

Rhoicosphenia abbreviata (AG.) LANGE-BERT.

Rhopalodia gibberula (EHR.) O. MULL. varheurckit

Synedra ulna (NITZ.) EHR. var oxyrhynchus ulna (NITZ.) EHR var. ulna

\section{ULOTHRYCOPHYCEES}

Ulothrix zonata KÜTZ.

Chaetophora sp.

EUCHLOROPHYCEES

Chlamydomonas sp.

\section{ZYGOPHYCÉES}

Closterium sp.

CYANOPHYCEES

Oscillatoria sp.

RHODOPHYCÉES

Audotinella sp.
Abréviations

H.f.

Ac.bio.

Ac. lan.

Ac. rost.

Ac. lap.

Ac. $\min$

Am. ped.

Co. pla.

Cy.af.

Cy. min.

Cy. $\sin$.

Cy. tum.

De, ten.

Di. hie.

Di. hie. mes.

Ep. ad.

Eu. pec.

F. cap.

F. rum.

F. vau.

F. vau, vau.

G. ang. pro.

G. dic.

G. trun.

Ha. arc.

Mel. cir.

Mer. cir.

Na. cry.

Na. lan.

Na. rhy.

Na. tri.

Ni. dis.

Ni. han.

Ni. lin.

Ni. pal.

Pi. bor.

Pi. vir.

Rh. abb.

Rh. gib.

Sy. uln. oxy.

Sy. uln. uln.

U.z.

Cha

Chla.

Clost.

Osc.

Aud. 
ELle renferme:

- des espèces fondamentales, définies par les plus fortes valeurs de fréquence centésimale 1 ( $50 \%$ ) et d'abondance relative ${ }^{2}(>5 \%)$ telles la Chrysophycée Hydrurus foetidus et les Diatomées Achnanthes minutissima, Hannaea arcus, Cymbella minuta et Gomphonema dichotomum;

- des espèces accompagnatrices, faiblement représentées (abondance relative < à $2 \%$ ), telles les Diatomées, Diatoma hiemale var. mesodon, Achnanthes bioreti, Cymbella sinuata, Navicula lanceolata, Achnanthes lanceolata et la variété rostrata, Achnanthes lapponica, Meridion circulare et l'Ulothricophycee Ulothrix zonata.

2) Un groupe constitué d'espèces à large amplitude écologique et dont l'abondance relative est comprise entre 0,1 et $10 \%$. Citons : Cocconeis placentula, Synedra ulna, Gomphonema augustatum var. productum, Fragilaria vaucheriae, Nitzschia palea et Navicula cryptocephala.

\section{2. - Répartition des taxons dans les différents microbiotopes}

Nous avons calculé la fréquence centésimale de chaque taxon pour chacune des faces des 20 cailloux prélevés (Tableau II).

Trois groupements d'espèces apparaissent (Tableau III) :

1) Un'groupe de 21 espèces, présentes sur toutes les faces du caillou mais n'ayant pas toutes la même fréquence :

- 16 Diatomées ont une fréquence supérieure à $45 \%$ (espèces constantes) sur les quatre microhabitats :

Achnanthes minutissima

A. bioreti

A. lanceolata

A. lapponica

Cocconeis placentula

Cymbella minuta

C. sinuata

Diatoma hiemale var. mesodon

1. Fréquence centésimale

Nombre de prélèvements où l'espece est présente

Nombre total de prélevements $\times 100$

2. Abondance relative

Nombre de cellules d'une espèce dans un prélèvement

Nombre total de cellules de ce prélèvement

$\times 100$
Fragilaria vaucheriae

Gomphonema augustatum var. productum

Gomphonema dichotomum

Hannaea arcus

Navicula cryptocephala

N. lanceolata

Nitzschia palea

Synedra ulna

-4 espèces ont une fréquence élevée ( $>50 \%$ ) uniquement au centre (B) et en aval (C) de la face supérieure ; elle est inférieure à $35 \%$ sur les deux autres microbiotopes (A et $\mathrm{D}$ ) : ce sont les diatomées Fragilaria capucina et Meridion circulare, la Cyanophycée Oscillatoria sp. et la Chrysophycée $H y d r$. rus foetidus (présente sous forme de thalle bien développé en $\mathrm{B}$ et $\mathrm{C}$ mais, uniquement à l'état de kystes, en $A$ et $D$ ).

- Une espèce de Rhodophycée Audouinella sp. est surtout fréquente en aval (40\%).

2) Un groupement, réunissant 10 taxons présents uniquement au centre (B) et en aval (C), est constitué par des algues filamenteuses assez fréquentes (> $50 \%$ ) Ulothrix zonata et Chaetophora sp. et 8 espèces de Diatomées dont seules Achnanthes rostrata, Fragilaria vaucheriae et Synedra ulna var. oxyrhynchus dépassent $50 \%$ en aval.

3) Un dernier groupe de 12 taxons, est cantonné uniquement en $\mathrm{C}$; ce sont des Diatomées pour la plupart. Parmi celles-ci, seule Fragilaria rumpens a une fréquence supérieure à $50 \%$ (espèce constante); les autres espèces, peu fréquentes $(F<30 \%)$ peuvent être considérées comme accidentelles (leur abondance relative est également faible $\mathrm{Ar}<1 \%$ ).

En définitive, seul le groupe des Diatomées, le plus diversifié, se répartit d'une façon assez équitable entre les différents microhabitats étudiés. Les algues vertes filamenteuses, ainsi que les autres groupes représentés sont surtout localisés en aval et au centre de la face supérieure de cailloux. Tous les taxons recensés en amont et sur la face inférieure sont retrouvés au niveau des autres faces et appartiennent au premier groupe (espèces présentes sur A, B, C, D).

\section{3. - Affinités coenotiques}

Afin d'apprécier les ressemblances entre les communautés d'algues des différents microbiotopes, le 
Tableau II. Fréquence centésimale des espèces algales déterminées pour chaque microhabitat des 20 cailloux.

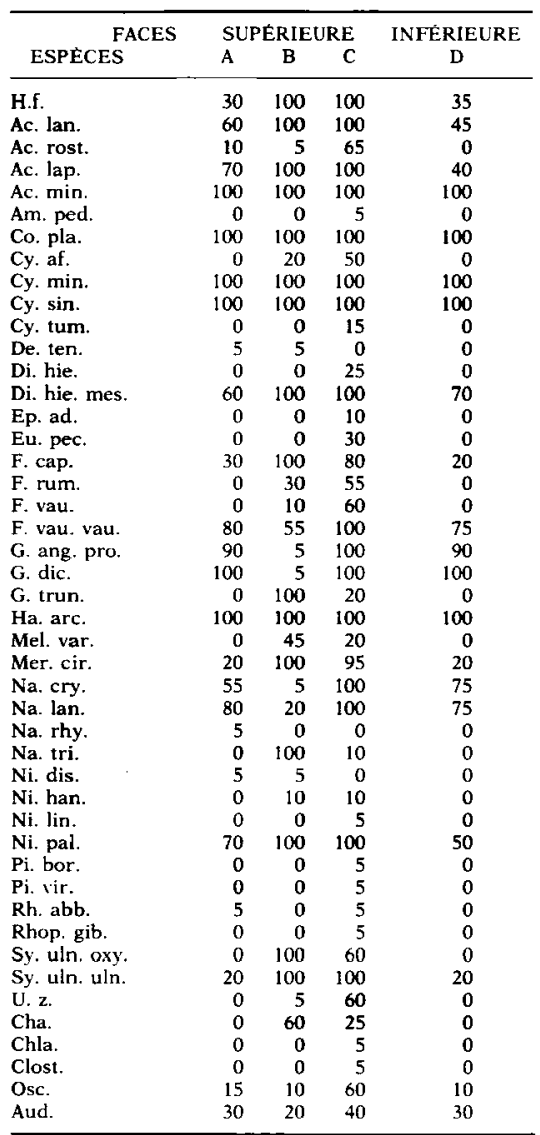

Tableau III. Répartition des taxons sur les 4 microhabitats.

\begin{tabular}{|c|c|c|c|}
\hline TAXONS & $\begin{array}{c}\text { Omni- } \\
\text { présents } \\
(\mathrm{A}, \mathrm{B}, \mathrm{C}, \mathrm{D})\end{array}$ & $\begin{array}{l}\text { Présents uni- } \\
\text { quement } \\
\text { en } B \text { et } C\end{array}$ & $\begin{array}{l}\text { Présents uni- } \\
\text { quement } \\
\text { en } C\end{array}$ \\
\hline H.f. & + & & \\
\hline Ac. lan. & + & & \\
\hline Ac. rost. & & + & \\
\hline Ac. lap. & + & & \\
\hline Ac. $\min$ & + & & \\
\hline Am. ped. & & & + \\
\hline Co. pla. & + & & \\
\hline Cy. af. & & + & \\
\hline Cy. min. & + & & \\
\hline Cy. sin. & + & & \\
\hline $\begin{array}{l}\text { Cy, tum. } \\
\text { De. ten. }\end{array}$ & & & + \\
\hline Di. hie. & & & + \\
\hline Di. hie. mes. & + & & \\
\hline Ep. ad. & & & + \\
\hline Eu. pec. & & & + \\
\hline F. cap. & + & & \\
\hline F. rum. & & & + \\
\hline F. vau. & & + & \\
\hline F. vau. vau. & + & & \\
\hline G. ang. pro. & + & & \\
\hline G. dic. & + & & \\
\hline G. trun. & & & \\
\hline Ha. arc. & + & & \\
\hline Mel. var. & & + & \\
\hline Mer. cir. & + & & \\
\hline Na. cry. & + & & \\
\hline Na. lan. & + & & \\
\hline Na. rhy. & & & \\
\hline Na. tri. & & + & \\
\hline Ni. dis. & & & e \\
\hline Ni. han. & & + & \\
\hline Ni. lin. & & & + \\
\hline Ni. pal. & + & & \\
\hline Pi. bor. & & & + \\
\hline Pi. vir. & & & \\
\hline Rh. abb. & & & \\
\hline Rhop. gib. & & & + \\
\hline Sy. uln. oxy. & & + & \\
\hline Sy. uln. uln. & + & & \\
\hline U. z. & & + & \\
\hline Cha. & & + & \\
\hline Chla. & & & + \\
\hline Clost. & & & + \\
\hline Osc. & + & & \\
\hline Aud. & + & & \\
\hline
\end{tabular}


coefficient d"association de Jaccard (1902) qui tient compte seulement de la présence des espèces, sans considérer leur abondance, a été utilisé.

La figure 2 montre les affinités coenotiques existant entre les autres microbiotopes analysés. Des affinités spécifiques élevées existent, d'une part, entre l'aval et le centre de la face supérieure et, d'autre part, entre l'amont de la face supérieure et le centre de la face inférieure.

Ces valeurs élevées traduisent, en effet, la présence sur les deux faces d'une communauté d'algues dont la composition en espèces est très voisine (Tableau III).
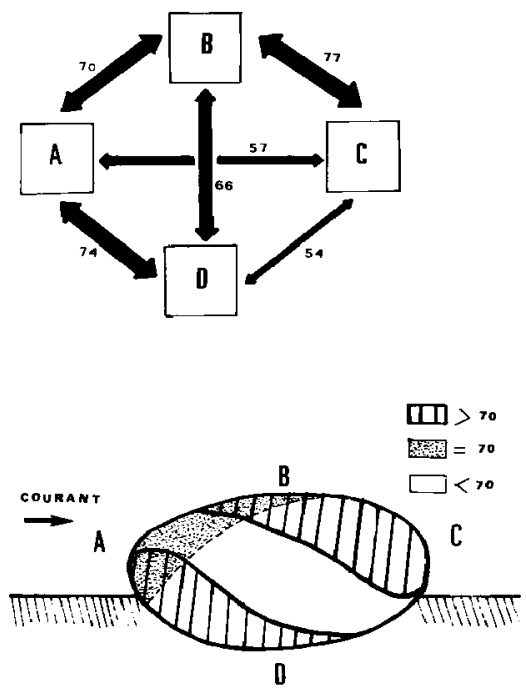

Fig. 2. Affinités coenotiques entre les microhabitats prospectés. Coefficients calculés d'après la méthode de Jaccard (1902); les valeurs indiquées correspondent aux moyennes arithmétiques.

\section{4. - Richesse spécifique et abondance moyennes des algues dans les microbiotopes}

\section{- RICHESSE SPÉCIFIQUE MOYENNE}

C'est la moyenne du nombre d'espèces déterminées sur chaque face du lot de 20 cailloux. Faible en $A$ et en $D$, elle augmente sur la face supérieure, de l'amont vers l'aval (de A en C) (fig. 3).

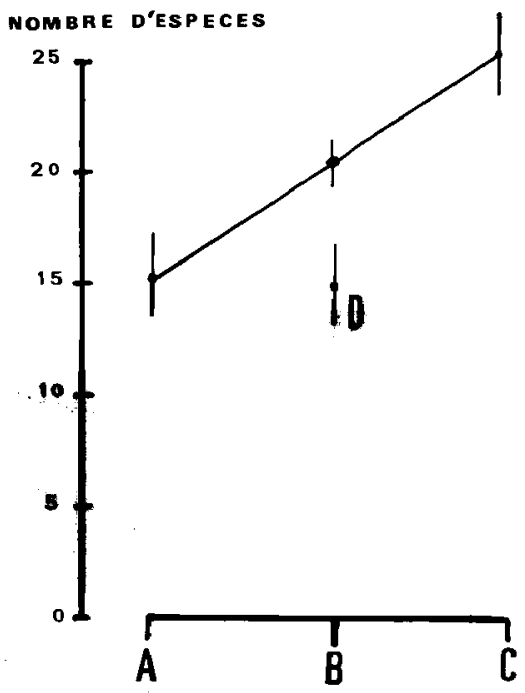

Fig. 3. Richesses spécifiques moyennes et amplitudes de variations au niveau des quatre microhabitats.

\section{- Densité algale moyenNe}

C'est la moyenne du nombre de cellules algales dénombrées sur chaque microhabitat du lot de 20 cailloux. Elle évolue exactement de la même manière que la richesse floristique; faible en $A$ et $D$, où elle est inférieure à $2500 / \mathrm{cm}^{2}$, elle atteint plus de $95.10^{3}$ cellules $/ \mathrm{cm}^{2}$ en C (fig. 4). En B, elle frôle $10^{4}$ cellules $/ \mathrm{cm}^{2}$.

\section{- Abondance relative moyenne des differents TAXONS}

C'est la moyenne des abondances relatives établies pour chaque espèce ou groupe taxonomique sur chaque face du lot de 20 cailloux.

En aval (C) et au milieu de la face supérieure (B), deux groupes d'algues dominent : les Chrysophycées 


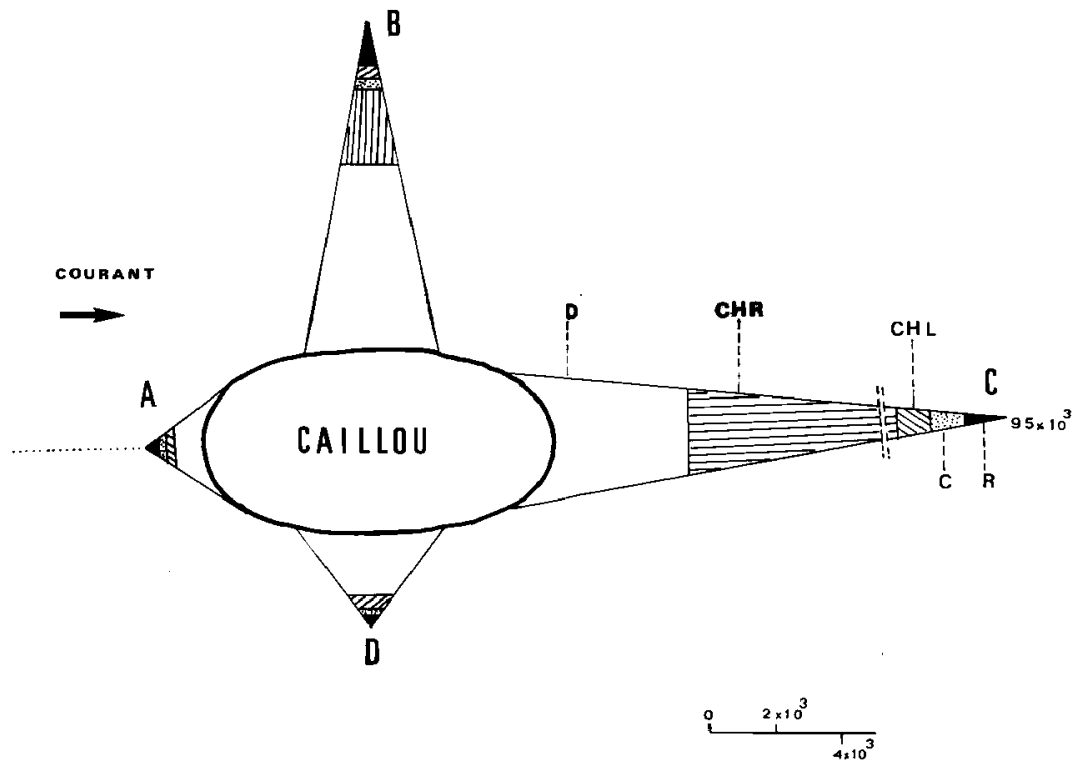

Fig. 4. Evolution de la densité algale moyenne et spectres floristiques des microhabitats. (La longueur de l'axe de symétrie du cône est fonction de l'effectif total exprimé en nombre de cellules par centimetre carré).

(53 et $34 \%$ ) et les Diatomophycées (44 et $61 \%$ ). Les Chrysophycées sont représentés par une seule espèce Hydrurus foetidus dont l'abondance constitue $53 \%$ du peuplement global en $C$ et $34 \%$ en B. Parmi les Diatomées, Achnanthes minutissima, Hannaea arcus, Cymbella minuta et Gomphonema dichotomum (fig. 5). Les Cyanophycées et les algues vertes filamenteuses sont faiblement représentées (respectivement 3 et $1 \%$ ).

Au niveau des autres microbiotopes, une seule classe, celle des Diatomophycées domine ( $85 \%$ ). Elle est essentiellement représentée par Achnantes minutissima, Hannaea arcus, Cymbella minuta, Gomphomema dichotomum et Cocconeis placentula (fig. 6). Les Cyanophycées ne dépassent pas $3 \%$ du peuplement algal tandis que les Chlorophycées ne sont pratiquement pas représentées.

\section{- Distribution des abondances}

Nous la représentons sous forme d'histogramme comprenant 5 classes d'abondance. Ils traduisent, de manière graphique, la structure du peuplement de chaque microbiotope.

Deux remarques s'imposent :

1) La distribution des abondances spécifiques est identique, d'une part entre les communautés de la face orientée vers l'aval et de la face supérieure et, d'autre part, entre les communautés de la face amont et de la face inférieure.

2) Les classes d'abondance caractérisant les deux modes de distribution sont généralement occupés par les mêmes taxons. 

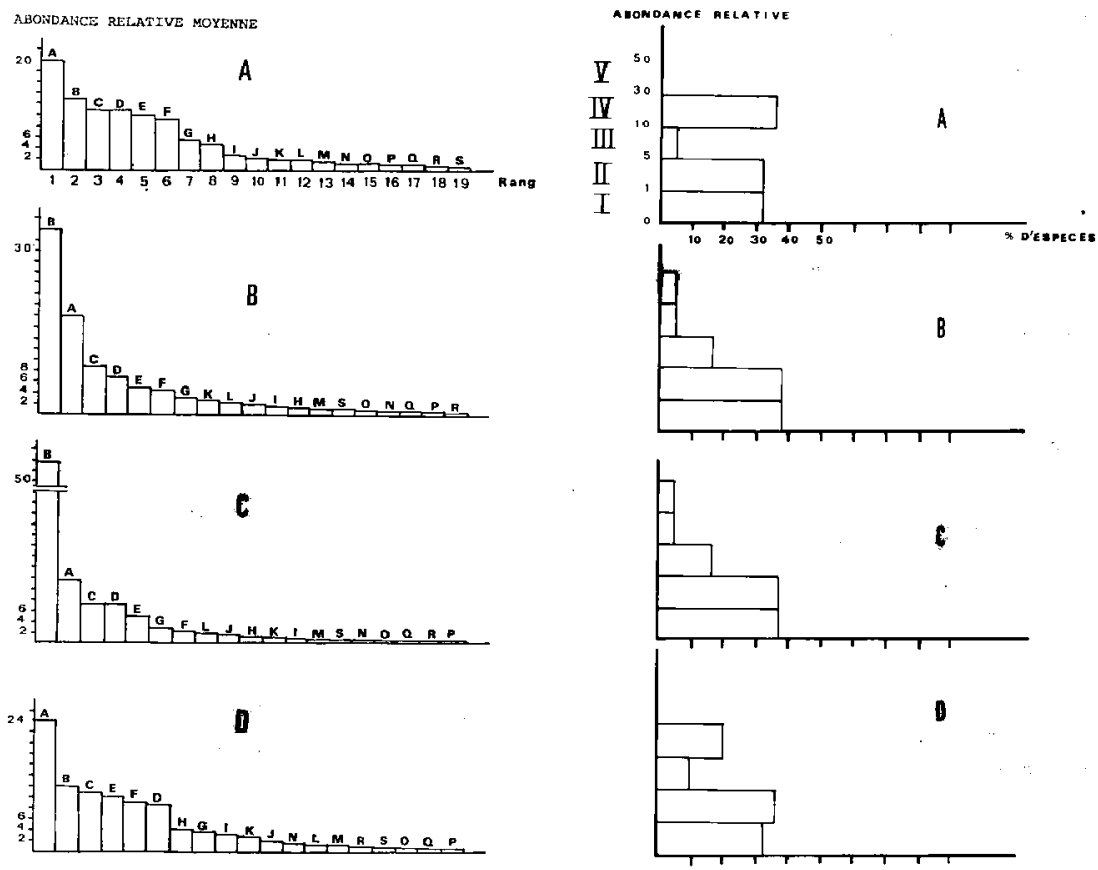

Fig. 5. Analyse en classe de rang des especes fréquentes et omniprésentes au niveau des quatre microhabitats (A, B, C, D).

Fig. 6. Distribution des classes d'abondance au niveau des quatre microhabitats. I : Ar $1 \% ;$ II : 1 ar 5; III : 5 Ar 10;IV: 10 Ar 30;V:30 Ar 50 .

1) Les zones d'eaux mortes, en aval des pierres, sont abritées et séparées de l'écoulement normal. A ce niveau, les pressions mécaniques du courant sont faibles.

2) Les zones à écoulement laminaire au contact des substrats à parois lisses (face postérieure) où s'éta. blit une mince couche d'eau (environ $1 \mathrm{~mm}$ ) à écoulement très ralenti (" couche limite ").

La distribution des algues sur les substrats naturels des eaux courantes et torrentueuses est, principalement, influencée par le courant, qui, par ses actions directes (mécaniques) ou indirectes (sur les 
autres caractéristiques physiques et microclimatiques locales), détermine le développement des microphytes :

1) En amont du caillou, l'action du courant limite le développement algal du fait de 2 influences : la force du courant lui-même et la projection des particules en suspension qu'il transporte. Les Diatomées, qui adhèrent au substrat, paraissent les mieux adaptées à ces conditions hydrologiques.

2) A la face supérieure du caillou, le ralent issement du courant dans la couche limite, permet le développement d'algues rhéophiles. Aux Diatomées, toujours dominantes, s'ajoutent des algues filamenteuses et thalloïdes.

3) En aval, zone d'eaux mortes, les algues filamenteuses et thalloïdes prolifèrent; la densité algale est la plus élevée. Ceci est en accord avec les observations de Mc Intire (1964).

4) A la face inférieure, le développement algal est faible. A ce niveau, l'action du courant est nulle et, la lumière pourrait être le facteur limitant.

\section{5. - Variabilité des résultats}

Trois paramètres statistiques ont été pris en compte : la moyenne, l'écart-type et le coefficient de variation qui exprime la variabilité des résultats obtenus (Heller 1968).

\subsection{1. - Fluctuations du nombre de taxons}

Tableau IV. Coefficient de variation calculé pour chaque microhabitat.

\begin{tabular}{|c|c|c|c|c|}
\hline & \multicolumn{3}{|c|}{ Face supérieure } & \multirow{2}{*}{$\begin{array}{c}\text { Face inférieure } \\
\text { Centre }\end{array}$} \\
\hline & Amont & Centre & Ava! & \\
\hline Moyenne & 16 & 19 & 25 & 15 \\
\hline Ecart-type & 2,82 & 0.70 & 4,66 & 2,83 \\
\hline $\begin{array}{l}\text { Coefficient } \\
\text { de variation } \Delta \%\end{array}$ & 17 & 4 & 18 & 19 \\
\hline
\end{tabular}

Le coefficient de variation ne dépasse pas $20 \%$ pour l'ensemble des microhabitats. Cependant, le fait important à noter, est la plus faible valeur enregistrée en $\mathbf{B}(4 \%)$; le nombre d'espèces (toujours les mêmes) recensées sur ce microbiotope varie donc très peu d'un caillou à l'autre. Sa richesse moyenne (19 espèces) s'est révélée très proche de la moyenne des richesses spécifiques globales (R.S.G.) qui concernent l'ensernble des 3 microhabitats d'un même caillou (Tableau V).

\subsection{2. - FluCtuATIONS DES DENSITES DES ESPECES ALGALES}

Pour analyser statistiquement les variations $\mathrm{du}$ nombre d'individus au niveau des quatre microbiotopes étudiés, 10 espèces omniprésentes ont été retenues suivant leur degré de densité décroissante dans le milieu.

- Espèces abondantes (abondance relative> $5 \%$ ) : Hydrurus foetidus, Achnanthes minutissima, Hannaea arcus et Cymbella minuta.

- Espèces peu abondantes ( $\mathrm{Ar}<5 \%$ ) : Cymbella sinuata, Navicula lanceolata, Achnanthes lanceolata var. lanceolata, Navicula cryptocephala, Achnanthes lapponica et Nitzschia palea.

a) Variations des densités absolues

Pour les quatre microbiotopes analysés, les résultats des dénombrements de chacune des 10 espèces omniprésentes et leur coefficient de variabilité, figurent dans les tableaux suivants. Ces coefficients de variabilité varient fortement pour toutes les espèces périphytiques. Cependant, les valeurs les plus faibles sont enregistrées en $B$, d'où l'intérêt de choisir cette zone pour réaliser l'échantillonnage.

b) Variations des abondances relatives des espèces algales

Nous avons calculé, dans chaque microbiotope, l'indice de variabilité pour les dix espèces omniprésentes (Tableau VII). Les valeurs les plus faibles sont encore enregistrées en $\mathrm{B}$; le pourcentage d'erreur est donc relativement plus faible à ce niveau.

En comparant ces valeurs avec les coefficients de variations établis pour les densités absolues (Tableau VI), on obtient, pour les espèces dominantes, une net te diminution de la variabilité en $\mathbf{B}$. Ainsi le coefficient de variation est de :
H. foetidus
A. minutissima
$55 \%$
$38 \%$
H. arcus
C. minuta
$91 \%$
$83 \%$
$71 \%$
$53 \%$
$88 \%$
$53 \%$
c) Variations des abondances relatives des différents groupes d'algues

Densité absolue Densité relative

Les pourcentages de variations les plus faibles sont obtenus en B (Tableau VIII) pour les deux groupes les mieux représentés du peuplement algal : les Diatomées et les Chrysophycées. 
Tableau V. Richeșsęs spécifiquęs globales (R.S.G.) calculées pour chaque cạillou.

$\begin{array}{llllllllllllllllllllllllll}\text { Ech. } & 1 & 2 & 3 & 4 & 5 & 6 & 7 & 8 & 9 & 10 & 11 & 12 & 13 & 14 & 15 & 16 & 17 & 18 & 19 & 20 & \mathrm{~m} & \mathrm{~s} & \%\end{array}$
$\begin{array}{llllllllllllllllllllllll}\text { R.S.G. } & 24 & 22 & 16 & 22 & 20 & 18 & 16 & 21 & 20 & 22 & 21 & 17 & 18 & 18 & 21 & 19 & 19 & 23 & 20 & 19 & 20 & 2,2 & 11\end{array}$

Tableau VI. Résultats des dénombrements des 10 algues périphytiques effectués sur les 4 microhabitats des cailloux.

H.f. Ac. min. Ha. arc. Cy. min. Cy. sin. Na. lan. Ac. lan. Na. cry. Ac. lap. Ni.pal.

FACE SUPERIEURE AMONT

$\begin{array}{lrrrrrrrrrr}m & 611 & 375 & 157 & 188 & 100 & 18 & 10 & 8 & 20 & 9 \\ \text { Ecart type } & 1320 & 414 & 134 & 162 & 145 & 16 & 10 & 9 & 34 & 8 \\ \Delta \% & 216 & 110 & 86 & 86 & 145 & 90 & 100 & 110 & 170 & 87\end{array}$

FACE SUPERIEURE CENTRE

$\begin{array}{lrrrrrrrrrr}m & 3182 & 2110 & 936 & 790 & 131 & 76 & 29 & 33 & 70 & 56 \\ \text { Ecart type } & 1760 & 1924 & 776 & 699 & 62 & 46 & 9 & 22 & 83 & 36 \\ \Delta \% & 55 & 91 & 83 & 88 & 48 & 61 & 32 & 66 & 118 & 64\end{array}$

FACE SUPERIEURE AVAL

\begin{tabular}{|c|c|c|c|c|c|c|c|c|c|c|}
\hline $\mathrm{m}$ & 67745 & 6883 & 4199 & 5835 & 365 & 304 & 66 & 98 & 227 & 215 \\
\hline Ecart type & 81133 & 7870 & 6073 & 7777 & 361 & 283 & 80 & 83 & 270 & 221 \\
\hline$\Delta \%$ & 119 & 123 & 144 & 133 & 98 & 93 & 121 & 84 & 118 & 102 \\
\hline \multicolumn{11}{|c|}{ FACE INFERIEURE } \\
\hline $\mathbf{m}$ & 764 & 475 & 194 & 194 & 51 & 25 & 15 & 12 & 8 & 7 \\
\hline Ecart type & 2408 & 663 & 294 & 314 & 61 & 39 & 33 & 16 & 11 & 9,6 \\
\hline$\Delta 0$ & 315 & 140 & 152 & 162 & 120 & 157 & 218 & 134 & 143 & 138 \\
\hline
\end{tabular}

Tableau VII. Abondances relatives des 10 espèces représentatives du peuplement, calculées au niveau des 4 microhabitats des cailloux.

\begin{tabular}{|c|c|c|c|c|c|c|c|c|c|c|}
\hline & H.f. & Ac. min. & Ha. arc. & Cy. min. & Cy. $\sin$. & Na. lan. & Ac. lan. & Na. cry. & Ac. lap. & Ni.pal. \\
\hline \multicolumn{11}{|c|}{ FACE SUPERIEURE AMONT } \\
\hline $\mathrm{m}$ & 13 & 20 & 11 & 11 & 5 & 1.3 & 0,76 & 0,5 & 1 & 0,6 \\
\hline Ecart type & 22 & 20 & 10 & 6,4 & 8 & 1,02 & 1 & 0,65 & 1 & 0.7 \\
\hline$\Delta \%$ & 172 & 60 & 89 & 59 & 163 & 78 & 132 & 130 & 100 & 116 \\
\hline \multicolumn{11}{|c|}{ FACE SUPERIEURE CENTRE } \\
\hline $\mathrm{m}$ & 34 & 18 & 9 & 7 & 1,5 & 1 & 0,5 & 0,5 & 0,9 & 0,8 \\
\hline Ecart type & 13 & 13 & 6 & 3,7 & 0,7 & 0,7 & 0,36 & 0,6 & 0,7 & 0,5 \\
\hline$\Delta \%$ & 38 & 71 & 73 & 53 & 49 & 69 & 72 & 132 & 75 & 59 \\
\hline \multicolumn{11}{|c|}{ FACE SUPERIEURE AVAL } \\
\hline $\mathrm{m}$ & 53 & 11 & 7 & 7 & 1,4 & 0,7 & 0,3 & 0,34 & 0,4 & 0,38 \\
\hline Ecart type & 24 & 6,7 & 6,9 & 4 & 1,5 & 0,7 & 0,5 & 0,5 & 0,4 & 0,46 \\
\hline$\Delta \%$ & 45 & 61 & 100 & 57 & 106 & 100 & 166 & 170 & 100 & 122 \\
\hline \multicolumn{11}{|c|}{ FACE INFERIEURE } \\
\hline $\mathrm{m}$ & 12 & 24 & 11 & 9 & 4 & 1,2 & 0,5 & 1 & 0,5 & 0,5 \\
\hline Ecart type & 10,6 & 16,2 & 11 & 6 & 4 & 1,7 & 0,75 & 1,5 & 0,66 & 0,8 \\
\hline$\Delta \%$ & 151 & 68 & 100 & 64 & 100 & 144 & 149 & 158 & 131 & 176 \\
\hline
\end{tabular}


Tableau VIII. Abondances relatives des différents groupes d'algues récoltées sur les 4 microhabitats des cailloux.

\begin{tabular}{|c|c|c|c|c|c|}
\hline & DIATOM. & CHRYSOP. & CYANOP. & CHLOROP. & RHODOP \\
\hline \multicolumn{6}{|c|}{ FACE SUPERIEURE AMONT } \\
\hline $\mathrm{m}$ & 85 & 13 & 1,4 & 0 & 0,5 \\
\hline Ecart type & 22 & 4 & 0 & 0 & 1,3 \\
\hline$\Delta$ & 26 & 170 & 296 & 0 & 261 \\
\hline \multicolumn{6}{|c|}{ FACE SUPERIEURE CENTRE } \\
\hline $\mathrm{m}$ & 61 & 34 & 3 & 1 & 0,5 \\
\hline Ecart type & 11 & 13 & 2,9 & 1,05 & 1,19 \\
\hline$\Delta$ & 11 & 38 & 96 & 105 & 238 \\
\hline \multicolumn{6}{|c|}{ FACE SUPERIEURE AVAL } \\
\hline m & 75 & 25 & 0 & 0 & 0 \\
\hline Ecart type & 22,6 & 23,4 & 1,8 & 2,47 & 0,96 \\
\hline$\Delta$ & 51 & 44 & 150 & 222 & 239 \\
\hline \multicolumn{6}{|c|}{ FACE INFÉRIEURE } \\
\hline m & 84.5 & 12 & 2,45 & 0 & 0,85 \\
\hline Ecart type & 21 & 19,5 & 9,8 & 0 & 2,74 \\
\hline$\Delta$ & 25 & 163 & 400 & 0 & 322 \\
\hline
\end{tabular}

b - Variations des abondances relatives des espèces algales

Nous avons calculé, dans chaque microbiotope, l'indice de variabilité pour les dix espèces omniprésentes (Tableau VIII). Les valeurs les plus faibles sont encore enregistrées en $B$; le pourcentage d'erreur est donc relativement plus faible à ce niveau.

En comparant ces valeurs avec les coefficients de variations établis pour les densités absolues (Tableau VII), on obtient, pour les espèces dominantes, une nette diminution de la variabilité en $\mathbf{B}$. Ainsi le coefficient de variation est de :

$55 \%$ en densité absolue et

$38 \%$ en densité relative pour $\quad H$. foetidus

$91 \%$ en denșité absolue et

$71 \%$ en densité relative pour A. minutissima

$83 \%$ en densité absolue et

$73 \%$ en densité relative pour $H$. Arcus

$88 \%$ en densité absolue et

$53 \%$ en densité relative pour

C. minuta

c - Variations des abondances relatives des différents groupes d'algues

Les pourcentages des variations les plus faibles sont obtenus en B (Tableau VIII) pour les deux groupes les mieux représentés du peuplement algal : les Diatomées et les Chrysophycées.
L'analyse de ces résultats montre que, plus une espèce, ou un groupe taxonomique domine le peuplement algal, plus le pourcentage d'erreur lié à son dénombrement est faible. Ainsi son abondance relative est, semble-t-il, beaucoup plus significative que sa densité absolue.

\subsubsection{COMPARAISON DE MOYENNE}

Afin d'apprécier les différences de densité et de richesse spécifique entre les 4 microhabitats, un test de comparaison de moyenne a été appliqué. Il en résulte :

- des différences non significatives entre les échantillons $A$ et $D$;

- des différences significatives entre, d'une part, les échantillons $B$ et, d'autre part, les échantillons A et D ;

- des différences hautement significatives entre les échantillons de l'aval (C) et ceux de A et $D$.

- des différences significatives entre $B$ et $C$.

\subsection{Cas particuliers}

Afin de connaitre l'influence de quelques caractéristiques physiques du substrat (exposition au courant, forme du caillou) sur la répartition des espèces, nous avons étudié la communauté algale de deux cailloux prélevés dans des conditions différentes. 
1 - Pour le caillou abrité derrière un gros bloc (environ $35 \mathrm{~cm}$ de hauteur), les résultats figurent dans le tableau ci-dessous :

\begin{tabular}{lrrrrr}
\hline & \multicolumn{5}{c}{ FACE SUPERIEURE FACE INFÉRIEURE } \\
mic rohabitat & \multicolumn{1}{c}{ A } & \multicolumn{1}{c}{ B } & \multicolumn{1}{c}{ C } & D \\
\hline $\begin{array}{l}\text { Uensité totale } \\
\text { Richesse } \\
\text { specifique }\end{array}$ & 21 & 16 & 1325 & 895 & 1180 \\
\hline
\end{tabular}

Nous avons comparé ces résultats avec ceux obtenus précédemment sur les 20 cailloux exposés directement au courant et qui présentaient la même organisation spatiale du peuplement. On constate une inversion de la colonisation du peuplement au niveau des microhabitats $\mathrm{A}$ et $\mathrm{C}$.

En A. partie du caillou abritée du courant (située en amont des eaux mortes du gros bloc), le peuplement algal est plus abondant. Il présente les mêmes caractéristiques qualitatives et quantitatives que celles observées au niveau de l'aval (C) des 20 cailloux.

En C, le peuplement est plus proche de celui de l'amont (A) des 20 cailloux. Ceci pourrait être expliqué par le degré d'agitation plus élevé produisant une turbulence intense à ce niveau (zone instable des eaux mortes, du gros bloc, selon Trivellato \& Décamps 1968).

2 - Pour le deuxième caillou, dont la face supérieure présente une cuvette centrale, on obtient le résultat suivant :

\begin{tabular}{|c|c|c|c|c|}
\hline & FACE & SLPER & IEURE & FACE INFĖRIEURE \\
\hline microhabitat & A & B & $\mathrm{C}$ & $\mathrm{D}$ \\
\hline \multirow{2}{*}{$\begin{array}{l}\text { densité totale } \\
\text { Richesse } \\
\text { spécifique }\end{array}$} & 38 & 55480 & 11220 & 260 \\
\hline & 8 & 32 & 26 & 13 \\
\hline
\end{tabular}

La répartition de la microflore sur les microha. bitats se fait selon le schéma général décrit précédemment. La composition du peuplement garde aussi le même aspect que sur les autres cailloux avec cependant, au niveau de la cuvette centrale, un développement algal plus important tant sur le plan qualitatif que quantitatif. En effet, une petite zone d'eaux mortes, créée au niveau de la dépression, permet la prolifération d'une microflore semblable à celle des eaux mortes de l'aval.
En définitive, les caractéristiques du milieu, liées soit à la position des supports dans le lit de la riviere (à l'arrière des gros blocs), soit à leur forme, conditionnent le mode de répartition du peuplement algal sur le caillou, sans pour autant modifier sa composition ou sa structure.

\section{4. - Discussion et conclusion}

L'étude des variations de la composition et de la structure du peuplement épilithique, en divers points d'un même caillou fait apparaître deux synusies, entité sociologique qui, selon la définition de Gisin (1947), " occupe l'espace d'un habitat limité ».

- la synusie 1, occupant la face orientée vers l'aval et le centre de la face supérieure du caillou.

- la synusie 2, occupant la face amont et le centre de la face inférieure.

En ce qui concerne le première synusie, les conditions hydrologiques particulières (influence de la couche limite, des eaux mortes...) favorisent l'installation d'une microflore diversifiée et représentative de la communauté des substrats caillouteux.

Les résultats de test statistiques montrent des différences notables, tant pour le nombre d'espèces que pour le nombre d'individus entre les différents microhabitats d'un même caillou. Ces différences sont hautement significatives entre les synusies 1 et 2 . Elles sont plus importantes également entre les microhabitats d'un même caillou que celles existant entre le même microhabitat de deux cailloux différents mais identiques dans leur forme et leur exposition.

Le peuplement de la face supérieure, du fait se sa plus grande stabilité est plus représentatif de la communauté algale des substrats caillouteux. Il présente :

- un plus grand nombre d'espèces caractéristiques ;

- une richesse spécifique moyenne voisine decelle du peuplement algal du cours d'eau (au niveau de la station) ;

- une densité algale élevée.

Les paramètres biotiques présentent des fluctuations plus importantes d'un caillou à l'autre au niveau du microhabitat de l'aval en relation probablement avec la complexité des conditions hydrodynamiques, au niveau des eaux mortes, qui s'établissent en arrière des cailloux. 


\section{Travaux cités}

Ambuhl (H.). 1959. -- Die Bedeutung der Strömung als ökologischer Faktor. Schweiz, Z. Hydrol, 21 ; 134-263.

Capblancq (J.) \& Cassan (M.). 1979. - Etude du périphyton d'une rivière polluée (]'Agout). I. Structure et développernent des communautés sur substrats artificiels. Annls Limnol., 15 (2) : 193.210.

Cassan (M.). 1978. - Etude du benthos de la rivière polluée Agout par la méthode des substrats artificiels : structure, croissance et métabolisme du périphyton. Thèse 3ème cycle, Univ. Toulouse: $135 \mathrm{p}$.

Castenholz (R.W.). 1961. - An evaluation of a submerged glass method of estimatif production of attached algae. Verh. Internat. Verein. Limmol., 14 (1): 155-159.

Cattaneo (A.) Ghittori (S.) \& Vendegna (V.) 1975. - The development of benthonic phytocoenosis on artificial substrates in the Ticino river. Oecologia, 19 (4) : 315-327

Cooper (J.M.) \& Wilhm (J.). 1975. - Spacial and temporal variation in productivity, species diversity, and pigment diversity of periphyton in a stream receiving domestic and oil refinery effluents South-west. Nat, 19 (4): 413-428

Coste (M.). 1978. - Sur I'utilisation des diatomées benthiques pour l'appréciation de la qualité biologique des eaux courantes. Méthodologie comparéc et approche typologique. Thèse Jème cycle, Univ. Franche-Comté, Besançon, 143 p.

Dauta (A.). 1978. - Colonisation de substrats artificiels dans la retenue de Malause. Cahiers du laboratoire d'hydrobiologie de Montereau, $7 ; 41-46$.

Décamps (H.), Capblancq (J.) \& Hirigoyen (J.P.). 1972. - Étude des conditions d'écoulement en canal expérimental. Verh. Internat. Verein. Limnol. $18: 718-725$.

Descy (J.P.). 1976. - Un appareillage pratique pour l'échantillonnage quartitatif du périphyton épilithique. Bull. Soc. ror. Bot Belg. 109 : 43-47.
Douglas (B.). 1958. - The ecology of the attached diatoms and other algac in a smail stony stream. J. Ecol., $46: 295-322$.

Galvin (N.). 1982. - Evolution d'une charge polluante dans un cours d'eau l'Argens (Var). Rôle d'un bief d'eau calme dans le Conctionnement du système. Thèse 3ème cycle, Univ. AixMarseille : $166 \mathrm{p}$

Galvin (N.) \& Cazaubon (A.). 1983. - Etude du periphyton d'un secteur pollué d'une rivière varoise l'Argens. Evolution spatiale du peuplement algal en période d'intense pollution. Annts Lim nol. 19 (3) : 169-178.

Gisin (H.). 1947. - Analyse et synthèse biocénotique. Arch. Sc. Phys. Nat. 5 (29) : $42-75$.

Heller (R.). 1968. - Manuel de statistique biologique, GautherVillars éd. Paris : $289 \mathrm{p}$.

Jaccard (P.). 1902. - Lois de distribution florale dans la zone alpine. Bull. Soc. Vaud. Sc. Nat. $38: 69130$.

Margalef (R.). 1956. - Algas de agua dulce del noreste de Espana. Publ. Inst. Biol. Apt. 22; 43-152.

Mc Intire (C.D.). 1964. - Some effects of current velocity on periphyton communities in laboratory streams. Hydrobiologia. $27: 559.570$

Neal (E.C.). Patten (B.C.) \& Depoe (C.E.). 1967. - Periphyton growth on artificial substrates in a radioactively contamined lake. Ecology, 48 (6) : 918-924.

Patrick (R.). 1973 - Use of algae, especially diatoms, in assesment of water quality. American Society for Testing and Materials. A.S.T.M. spec. Tech. Publ., 528 : 76-95.

Trivellato (D.) \& Decamps (H.). 1968. - Influence de quelques obstacles simples sur l'écoulement dans un Ruisseau Expérimental. Annls Limnol 4 (3) : 357-386.

Young (OW.). 1945. - A limnological investigation of periphyton in Douglas Lake, Michigan. Trans. Amer. Micr. Soc., 64 : 1-20. 